\title{
EUS is superior to secretin-enhanced cholangio-MRI to establish the etiology of idiopathic acute pancreatitis
}

\section{다)(웅}

\section{Authors}

Juan J. Vila', F. Javier Jiménez Mendioroz², Paul Yeaton² ${ }^{3}$ Iñaki Fernández-Urién', José Luis García Sanchotena², Silvia Goñi ${ }^{1}$, Marta Gómez Alonso ${ }^{1}$, Juan Carrascosa ${ }^{1}$, Ana Borda ${ }^{1}$, Carlos Prieto ${ }^{4}$, Jesús Urman ${ }^{4}$, José M Zozaya ${ }^{1,4}$

Institutions

1 Endoscopy Unit, Gastroenterology Dpt, Complejo Hospitalario de Navarra, Pamplona, Spain

2 Digestive Radiology Unit, Radiology Dpt, Complejo Hospitalario de Navarra, Pamplona, Spain

3 Endoscopy Unit, Gastroenterology Department, Carilion Clinic, Roanoke, Virginia, United States

4 Biliary and Pancreatic Diseases Unit, Gastroenterology Dpt, Complejo Hospitalario de Navarra, Pamplona, Spain

submitted 29.11.2019

accepted after revision 8.7.2020

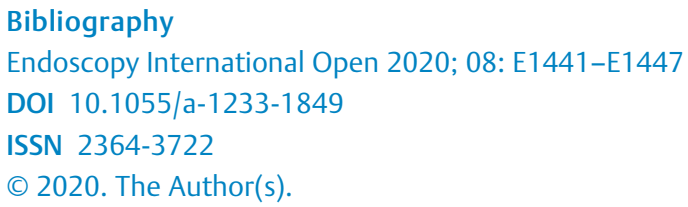

This is an open access article published by Thieme under the terms of the Creative Commons Attribution-NonDerivative-NonCommercial License, permitting copying and reproduction so long as the original work is given appropriate credit. Contents may not be used for commecial purposes, or adapted, remixed, transformed or built upon. (https://creativecommons.org/licenses/by-nc-nd/4.0/)

Corresponding author

Juan José Vila Costas, c| Irunlarrea, 3. Postal code: 31.008

Pamplona, Spain

Fax: +848422303

juanjvila@gmail.com

\section{ABSTRACT}

Background and study aims The etiology of idiopathic acute pancreatitis (IAP) should always be defined. Our aim was to compare the diagnostic value of endoscopic ultrasound (EUS) versus secretin-enhanced magnetic resonance cholangiopancreatography (S-MRCP) in patients with IAP. Patients and Methods Patients admitted to a single tertiary care University hospital with IAP were invited to participate in the study. Enrolled patients underwent EUS and SMRCP in a single-blinded comparative study. EUS and SMRCP were performed no sooner than 4 weeks after discharge. The diagnostic yield of EUS and S-MRCP and demographic variables were included in the analysis. Additional follow-up, results of subsequent serology, radiographic exams, and relevant histological analysis were considered in determination of the final diagnosis.

Results A total of 34 patients were enrolled; EUS was normal in six, cholelithiasis was defined in 15 , choledocholithiasis in two, pancreas divisum in three, branch-type intraductal papillary mucinous tumor (IPMT) in three, and chronic pancreatitis in five. S-MRCP identified choledocholithiasis in one, divisum in four, branch-type IPMT in three, chronic pancreatitis in two; 24 subjects diagnosed as normal by S-MRCP. Diagnostic correlation between EUS and S-MRCP was slight (kappa $=0.236$, $95 \%$ confidence interval: $0.055-0.416)$. EUS provided a statistically significantly higher diagnostic yield than S-MRCP: $79.4 \%$ (CI95\%: $65 \%-94 \%)$ vs $29.4 \%$ (CI95\%: $13 \%-46 \%)(P=0.0002)$. The sensitivity, specificity, and positive and negative predictive values of EUS and S-MRCP were $90 \%, 80 \%, 96 \%, 57 \%$ and $33 \%, 100 \%, 100 \%$ and $16 \%$, respectively.

Conclusion The diagnostic yield of EUS is higher than SMRCP in patients with IAP.

\section{Introduction}

Identifying the cause of acute pancreatitis represents a diagnostic challenge in a significant number of patients. Diagnostic etiology is undefined in $10 \%$ of patients with a first episode of acute pancreatitis and in $30 \%$ with recurrent acute pancreatitis. The initial evaluation should include history of infectious dis- ease, trauma, or recent abdominal surgery; alcohol and drug intake; calcium analysis, lipid profile, ANA and IgG4. Imaging typically includes transcorporeal abdominal ultrasound and possibly cross-sectional imaging. If the cause is not defined following these exams, patients can be considered to have idiopathic acute pancreatitis (IAP) [1-3]. 
Identifying the cause of pancreatitis in patients with IAP is important, because up to $70 \%$ may suffer from recurrent episodes [4], and increased mortality has been described in these individuals [5]. Endoscopic retrograde cholangiopancreatography (ERCP) has been considered as part of the diagnostic pathway, reporting a diagnostic yield reaching up to $80 \%$, but with $10 \%$ to $15 \%$ incidence of adverse events (AEs) [6,7] including post-ERCP pancreatitis. Another technique is collection of bile for microscopic bile examination (MBE) with a polarized filter. This has a false-negative rate of $29 \%$ to $50 \%$, is time consuming, and cannot be completed in up to $20 \%$ of patients [8, 9].

Given the disadvantages of ERCP and MBE, two techniques have been demonstrated to have a high diagnostic value in IAP: endoscopic ultrasonography (EUS) and magnetic resonance cholangiopancreatography (MRCP). EUS provides an etiologic diagnostic yield of $60 \%$ to $80 \%[3,10-14]$. The yield of MRCP ranges from $32 \%$ to $90 \%$ and improves when associated with secretin stimulation (S-MRCP) $[15,16]$.

The diagnostic yield of both examinations in patients with IAP has been compared in three studies with variable results that have not demonstrated the superiority of either modality [17-19]. In two studies, EUS was compared with MRCP with no secretin stimulation $[18,19]$. In the third study, ERCP was included in the comparison performed in patients with recurrent IAP [17].

Considering this background, we proposed a unique study comparing the diagnostic yield between EUS or with S-MRCP in patients with IAP.

\section{Patients and methods}

We designed a prospective, single-blind, comparative study, offering enrollment to consecutive patients admitted with IAP to a single tertiary University hospital between January 2009 and December 2013; patients were followed for a minimum of 6 months.

Acute pancreatitis was defined as the occurrence of abdominal pain associated with amylase and lipase levels three times and twice over normal value, respectively. We considered IAP when there was no recent history of infection, trauma or surgery; no history of alcohol consumption $>60 \mathrm{~g}$ of ethanol per day in men and $>40 \mathrm{~g}$ for women, or drugs implicated in pancreatitis; IgG4 and ANA were normal; triglycerides $<500 \mathrm{mg} / \mathrm{dL}$, calcium $<10 \mathrm{mg} / \mathrm{dL}$; and two abdominal ultrasound or an ultrasound and computed tomography scan not showing cause of acute pancreatitis.

Patients who met these criteria were diagnosed with IAP and were invited to participate in the study. The inclusion criteria in this study were age $>18$ years, diagnosis of IAP during the period of inclusion, willingness to undergo EUS and S-MRCP, and willingness to be included in the study after the informed consent process.

Patients with Billroth II or total gastrectomy were excluded because the study with EUS could not be completed.

The included patients underwent EUS and S-MRCP no sooner than 4 weeks after hospital discharge for IAP. Examinations were performed by two echoendoscopists and two radiologists, each of whom had a minimum of 3 years of experience.

After each EUS and S-MRCP was performed, a report was delivered to the control researcher. The control researcher was responsible for recording the patients included, scheduling examinations, and recording EUS and S-MRCP results. After both reports were received, the researcher recorded them in the electronic medical record, making them available to the physicians involved in clinical care. The echoendoscopists and radiologists performing the examinations were thus blinded to the results of the other examination.

Data recorded for analysis included information on demographics, prior cholecystectomy, prior episodes of pancreatitis, EUS and S-MRCP findings, and any treatments performed. During the minimum 6-month follow-up period, clinical outcomes and results of new examinations performed were included in defining the final diagnosis.

EUS exams were performed with a radial echoendoscope (EG3670URK, Pentax, Hamburg, Germany). Cholelithiasis and choledocholithiasis were defined by an arch-shaped image with posterior acoustic shadowing in gallbladder or bile duct. Microlithiasis was defined by the occurrence of fleeting, unshaded, echogenic, point-shaped particles in the gallbladder. Given the clinical implication being identical, the presence of microlithiasis or gallstones were defined as cholelithiasis. We considered a minimum of five diagnostic criteria defined by Sahai [20] to establish a diagnosis of chronic pancreatitis.

S-MRCP was performed using a 1.5 Tesla magnet (Magnetom Avanto, Siemens, Erlangen, Germany) with the patient in a fasting state. The following parameters were employed: TR110, TE4,72, G6 mm for axial T1; TR1000, TE85, G6 mm for axial HASTE and TR4500, TE754, G30 mm with apnea for a heavily T2-weighted cholangiographic sequence with/without secretin. Secretin $0.1 \mathrm{U} / \mathrm{kg}$ was injected as a bolus, with images obtained every minute for 10 minutes after injection. Five minutes before the S-MRCP, 400 cc of negative oral contrast was administered. Pancreatic ductal and parenchymal morphology, gallbladder or ductal stones, reduced T1 signal intensity, pancreatic secretion, and ductal morphology were evaluated before and after secretin injection as well as response of the sphincter of Oddi. Chronic pancreatitis was diagnosed based on ductal findings according to the Cambridge classification [21].

For the diagnosis of cholelithiasis, we considered the EUS result as definitive. During the follow-up period, some patients underwent cholecystectomy and ERCP. The final diagnosis of cholelithiasis was further refined by these interventions. Likewise, a final diagnosis of chronic pancreatitis may have included data derived from subsequent ERCP and cross-sectional imaging. A final diagnosis of pancreas divisum included subsequent ERCP in some patients. Some patients with pancreatic cystic lesions underwent subsequent resection to refine the final diagnosis. To establish a final diagnosis, we considered all the study results, examinations, and interventions in the follow-up period. Therapeutic decisions were made based on clinical indications by a multidisciplinary team including clinicians, endoscopists, radiologists, and surgeons. 
- Table 1 Demographic, clinical and radiological data of the 34 patients before inclusion in the study.

\begin{tabular}{|l|l|}
\hline Male/female & $17 / 17$ \\
\hline Age & $63.53 \pm 12.63$ (range: $21-78$ years) \\
\hline Abdominal ultrasound & $2.21 \pm 1.51$ (range: $1-7)$ \\
\hline Abdominal CT & $1.18 \pm 1.31$ (range: $0-5)$ \\
\hline Previous pancreatitis & $1.52 \pm 1.06$ (range: $1-5)$ \\
\hline Previous cholecystectomy & 7 patients \\
\hline CT, computed tomography & \\
\hline
\end{tabular}

Statistical analysis was performed with SPSS 20. Quantitative descriptive data are provided as mean \pm standard deviation and range. The degree of accordance between EUS and S-MRCP was evaluated by using the kappa statistic. Sensitivity, specificity, and positive and negative predictive values were estimated to study diagnostic test validity. In addition, to compare the number of successes between EUS and S-MRCP, success rates were estimated with confidence intervals at $95 \%$, and the McNemar test for paired samples was used. $\mathrm{P}<0.05$ was considered statistically significant.

The study was approved by our hospital scientific research ethics committee.

\section{Results}

All 40 patients admitted with IAP during the study period were invited to enroll; four refused EUS and two had a pacemaker, which precluded S-MRCP. Demographic, clinical, and radiological data from the 34 patients finally enrolled are shown in $>$ Table 1.

The mean time between performance of EUS and S-MRCP was 13.82 \pm 13 days (range: 2-49). Etiologic findings of EUS and S-MRCP compared with the final diagnosis are shown in - Table 2. In four patients, a possible second cause was found on EUS that was not considered responsible for IAP. Patients were followed up for a mean of $31.62 \pm 19.20$ months (range: 6-60) after EUS and S-MRCP.

Thirteen of 15 patients with cholelithiasis on EUS underwent cholecystectomy. Gallbladder histological examination showed chronic cholecystitis in 12 patients, associated with cholesterol polyps in three and gallbladder adenomyosis in one. The other patient had macroscopically visible stones smaller than $3 \mathrm{~mm}$ in the gallbladder. During follow-up, one of these patients suffered recurrent pancreatitis. Another patient developed abdominal pain 2 years after IAP and 1 year later was diagnosed with unresectable pancreatic cancer. One patient who was asymptomatic after cholecystectomy suffered sudden death after 1 year of follow-up. Three patients refused cholecystectomy and remained asymptomatic.

The initial diagnosis of chronic pancreatitis made in EUS was confirmed during follow-up in four of five patients by means of subsequent EUS and functional testing. One patient suffered recurrent episodes of pancreatitis and the other three remained asymptomatic. In the fifth patient, chronic pancreatitis

\begin{tabular}{|c|c|c|c|c|c|c|c|c|}
\hline & & \multicolumn{6}{|c|}{ Final Diagnosis } & \multirow[t]{2}{*}{ Total } \\
\hline & & Normal & Cholelithiasis & CBD Stones & Pancreas Divisum & IPMT & $\mathrm{CP}$ & \\
\hline \multirow[t]{6}{*}{ EUS } & Normal & 4 & & & 1 & 1 & & 6 \\
\hline & Cholelithiasis & & 15 & & & & & 15 \\
\hline & CBD stones & & & 2 & & & & 2 \\
\hline & Pancreas divisum & & & & 3 & & & 3 \\
\hline & IPMT & & & & & 3 & & 3 \\
\hline & $\mathrm{CP}$ & & 1 & & & & 4 & 5 \\
\hline \multirow[t]{6}{*}{ S-MRCP } & Normal & 4 & 16 & 1 & 0 & 1 & 2 & 24 \\
\hline & Cholelithiasis & & & & & & & 0 \\
\hline & CBD stones & & & 1 & & & & 1 \\
\hline & Pancreas divisum & & & & 4 & & & 4 \\
\hline & IPMT & & & & & 3 & & 3 \\
\hline & $\mathrm{CP}$ & & & & & & 2 & 2 \\
\hline Total & & 4 & 16 & 2 & 4 & 4 & 4 & \\
\hline
\end{tabular}






- Fig. 1 Diagnostic, therapeutic, and follow-up etiological data from patients with IAP included in the study.

was not confirmed in a subsequent EUS where biliary microlithiasis was observed. This patient was considered a false-positive in EUS for chronic pancreatitis and a false-negative for cholelithiasis in the initial EUS.

The two patients with choledocholithiasis underwent ERCP, which confirmed the diagnosis. Both remained asymptomatic thereafter. In addition, three of four patients with pancreas divisum underwent ERCP with minor papilla sphincterotomy and stent placement. One subsequently suffered another episode of pancreatitis. The fourth patient refused ERCP and remained asymptomatic.

One patient with intraductal papillary mucinous tumors (IPMT) showed signs of malignancy on EUS with fine-needle aspiration, which were not identified on S-MRCP. Malignancy was confirmed histologically after surgical resection and the patient remained asymptomatic thereafter. Side-branch IPMT was also confirmed histologically in two more patients after surgical resection. One patient suffered recurrence of pancreatitis. The fourth patient did not undergo surgery because of high surgical risk and the cyst became malignant and the patient died 3 years after diagnosis. The size of these IPMTs ranged between $5 \mathrm{~mm}$ and $40 \mathrm{~mm}$ and were located in the uncinate process (1), pancreatic head (2) and pancreatic body (1).

In summary, the final diagnoses in this series were cholelithiasis in 16 patients, choledocholithiasis in two, pancreas divisum in four, chronic pancreatitis in four and side-branch IPMT in four patients ( $\triangleright$ Table 2 ). In the remaining four patients, the cause of pancreatitis was not identified with EUS or S-MRCP or during follow-up. The diagnostic, therapeutic and follow-up data from the patients included are described in $>$ Fig. $\mathbf{1}$.

According to these final results, a correct etiologic diagnosis was made in 27 patients (79.4\%) with EUS and in 10 (29.4\%) with S-MRCP. Nineteen of 24 patients with normal findings on S-MRCP were diagnosed with EUS. Two of six patients with normal EUS were diagnosed with S-MRCP.

According to the kappa correlation value, a slight agreement between EUS and S-MRCP was found (kappa=0.236, SE of kappa $=0.092$, $95 \%$ confidence interval: 0.055-0.416). The diagnostic value of EUS was statistically higher than S-MRCP: 79.4\% (Cl 95\%: 65\%-94\%) vs $29.4 \%(\mathrm{Cl} 95 \%$ : $13 \%-46 \%)(\mathrm{P}=$ 
$0.0002)$. The sensitivity, specificity, and positive $p$ and negative predictive values of EUS and S-MRCP for the etiologic diagnosis of IAP were $90 \%, 80 \%, 96 \%, 57 \%, 33 \%, 100 \%, 100 \%$, and $16 \%$, respectively. In patients with gallbladder $(n=27)$, the difference in the diagnostic value between EUS and S-MRCP was increased: $81 \%$ (Cl 95\%: 66-97\%) of patients were diagnosed with EUS compared to $19 \%$ (CI 95\%: 3-34\%) with S-MRCP (P= $0.0007)$. In cholecystectomized patients $(n=7)$, the diagnostic difference disappeared and both examinations were diagnostic in five patients $(P=1)$.

\section{Discussion}

In this study, the value of EUS was significantly higher than that of S-MRCP in the etiologic diagnosis of patients with IAP. The diagnostic values of EUS and MRCP in patients with IAP have been compared prospectively in three studies [17-19]. The first study compared the diagnostic yield of S-MRCP, secretin-enhanced EUS and ERCP in 44 patients with recurrent IAP [17]. The diagnostic values were $65.9 \%, 79.5 \%$, and $62.8 \%$ respectively $(P>0.05)$.

The second study compared the diagnostic value of EUS and MRCP in 49 patients with IAP [18]. The diagnostic yield of EUS was statistically significantly higher ( $51 \%$ vs $20 \%$; $P=0.001)$, especially in patients with gallbladder ( $60 \%$ vs $11 \%$; $\mathrm{P}=0.008$ ) irrespective of the number of pancreatitis bouts.

The third study described the diagnostic yield of both examinations within a comprehensive diagnostic algorithm for acute pancreatitis [19]. In this study, 128 patients were enrolled and an etiologic diagnosis was found on initial examination in 83 (65\%). Thirty-eight of the remaining 45 patients underwent EUS and MRCP, showing a higher diagnostic yield with EUS (39.5\% vs $21 \%$ ) without statistically significant differences ( $P=$ $0.09)$.

There are important methodological differences in our study compared with the previous three studies regarding population included, design, examinations performed, and timing of the examinations. The sample of patients included in our study is similar those of Repiso et al [18] and Thevenot et al [19]. However, Mariani et al [17] included a highly selected sample of patients with recurrent IAP with normal bile duct. These patients represent $<32 \%$ of the sample of the other studies.

Moreover, although these studies are prospective and blinded, none explain how this condition was achieved. In our study a control researcher kept the result of each examination unavailable until both were performed. Besides, we wanted to guarantee that the study design did not harm the patient ethically by delaying the diagnostic or therapeutic process.

There are also differences in the examinations performed. Mariani et al [17] added stimulation with secretin to EUS, a technique not widely used in clinical practice, limiting the applicability of the results [18]. Repiso et al [18] and Thevenot et al [19] performed MRCP without secretin stimulation, unlike our study.

With regard to the timing of examinations, Thevenot et al [19] performed most EUS and MRCP on the same day. Repiso et al [18] performed the EUS first, with a mean time difference of $24 \pm 15$ days with regard to the MRCP, and Mariani et al [17] did not report this information. This time difference may be important because in $40 \%$ of patients, biliary sludge disappears completely, a further $40 \%$ show a cyclical pattern of occurrence, and in $20 \%$, there is progression to cholelithiasis [2, 22, 23]. Therefore, the diagnosis of biliary microlithiasis may vary depending on the timing with regard to the IAP bout. Although in our study was not possible to perform both examinations on the same day for logistical reasons, we tried to reduce this variability as much as possible. The mean time difference between both examinations was $13.82 \pm 13$ days (range: $2-49$ ), lower than with Repiso et al [18].

The most frequent finding in our study, as in that of Repiso et al [18] and Thevenot et al [19], was microlithiasis or biliary cholelithiasis. This finding makes the difference between EUS and S-MRCP, because no cases of biliary microlithiasis were diagnosed with it, as in prior studies [17-19]. Furthermore, it is the most common cause of IAP in most studies, with a incidence as high as $80 \%[2,24]$. Although MBE has been considered the gold standard for diagnosing biliary microlithiasis, more recent studies have shown better results with EUS [25, 26 ] and justify the use, along with other authors [17], of this result as a baseline in our study. In addition, the finding of chronic cholecystitis has been associated with the presence of gallstones [27, 28]. In our study, 12 of 13 patients undergoing cholecystectomy had chronic cholecystitis, which supports the diagnosis obtained by EUS.

However, in the study by Mariani et al [17], the most frequent finding was chronic pancreatitis. This is related to the high sample selection, but also to the diagnostic criteria considered to diagnose IAP and chronic pancreatitis. In our study, alcohol consumption $>60 \mathrm{~g}$ in men and $>40 \mathrm{~g}$ in women was considered to be a possible cause of pancreatitis and we diagnosed chronic pancreatitis with five EUS criteria. This cutoff point provides sensitivity and specificity of $60 \%$ and $83 \%$, respectively, a high positive predictive value, an excellent correlation with the ERCP for moderate and severe involvement $(\mathrm{k}=0.82)$, and a good interobserver correlation $(\kappa=0.45)[20,29]$. Repiso et al [18] placed the limit at $80 \mathrm{~g}$ of ethanol and three diagnostic criteria. Thevenot et al [19] limited consumption to $40 \mathrm{~g}$ and did not specify the number of EUS diagnostic criteria for diagnosis of chronic pancreatitis. Mariani et al [17] stated that patients who did not abuse alcohol were enrolled and the diagnostic cutoff was four criteria. The percentage of patients diagnosed of chronic pancreatitis clearly varies depending on these parameters. In our study and that of Thevenot et al [19], chronic pancreatitis was diagnosed in $11.8 \%$ and $10.5 \%$ of patients while Mariani et al [17] and Repiso et al [18], with less restrictive criteria, report a higher percentage of $25 \%$ and $18 \%$, respectively.

The diagnostic yield of MRCP in our study was $29.4 \%$, higher than that of Repiso et al (20\%) [18] and Thevenot et al (21\%) [19]. This difference is explained by the use of S-MRCP in our study, which improves results of MRCP [16]. The diagnostic yield of S-MRCP in the study by Mariani et al [17] was significantly higher (65.9\%), which again can be explained by the 
highly selected sample of the patients enrolled and a lower restriction on the alcohol intake limit.

Our study has various limitations. First, the study sample was heterogeneous because patients with and without gallbladder and with a single incident of and recurrent IAP were enrolled. Because of the small sample size, no subpopulation analysis could be performed, which may be regarded as a second limitation. Third, although we tried to minimize the time gap between EUS and S-MRCP, medical reasons or intercurrent processes delayed the second examination in some cases, increasing the difference. Finally, taking the result of the baseline EUS and the S-MRCP into consideration may give rise to diagnostic bias. However, EUS is currently considered the gold standard for diagnosis of chronic pancreatitis and MRCP the gold standard for pancreas divisum, and no examination offers better results for diagnosing pancreatic cystic lesions than these two methods. To reduce this diagnostic bias, the histological results and the follow-up data were considered.

On the other hand, the prospective, single-blinded design and use of a control researcher, thereby minimizing biases, are remarkable strengths of the study. Second, comparison of EUS with S-MRCP, which is the best version of MRCP, was made. Finally, the etiological data from long-term follow-up were considered definitive. As a result, the initial diagnostic yield of EUS declined as a patient's etiological diagnosis changed. This reduction in the initial diagnostic yield during follow-up has been described previously [12].

\section{Conclusion}

In conclusion, in our study, the diagnostic value of EUS was significantly higher than that of S-MRCP in patients with IAP. In our opinion and according to our results, a patient with IAP and a gallbladder should undergo EUS as the first examination to search for etiologic diagnosis. If the cause remains unidentified after EUS, S-MRCP should be performed. Further studies with adequate samples should clarify the role of both examinations in cholecystectomized patients with IAP.

\section{Competing interests}

Dr. Vila is a consultant for Boston Scientific

\section{References}

[1] Steinberg W, Tenner S. Acute pancreatitis. N Engl J Med 1994; 330 : $1198-1210$

[2] Lee SP, Nicholls JF, Park HZ. Biliary sludge as a cause of acute pancreatitis. N Engl J Med 1992; 326: 589-593

[3] Vila JJ. Endoscopic ultrasonography and idiopathic acute pancreatitis. World J Gastrointest Endosc 2010; 2: 107-111

[4] Bank S, Indaram A. Causes of acute and recurrent pancreatitis. Clinical considerations and clues to diagnosis. Gastroenterol Clin North Am 1999; 28: 571-589, viii
[5] Kohut M, Nowak A, Nowakowska-Duława E et al. The frequency of bile duct crystals in patients with presumed biliary pancreatitis. Gastrointest Endosc 2001; 54: 37-41

[6] Mallery JS, Baron TH, Dominitz JA et al. Complications of ERCP. Gastrointest Endosc 2003; 57: 633-638

[7] Coyle W], Pineau BC, Tarnasky PR et al. Evaluation of unexplained acute and acute recurrent pancreatitis using endoscopic retrograde cholangiopancreatography, sphincter of Oddi manometry and endoscopic ultrasound. Endoscopy 2002; 34: 617-623

[8] Moskovitz M, Min TC, Gavaler JS. The microscopic examination of bile in patients with biliary pain and negative imaging tests. Am J Gastroenterol 1986; 81: 329-333

[9] Reyes López A, Miño Fugarolas G, Costán Rodero G et al. Value of duodenal drainage in the etiologic diagnosis of acute pancreatitis. Rev Esp Enferm Dig 1993; 83: 363-366

[10] Govil A, Agrawal MK, Agrawal D et al. Role of endoscopic ultrasonography in patients with first episode of idiopathic acute pancreatitis. Indian J Gastroenterol 2014; 33: 241-248

[11] Morris-Stiff G, Al-Allak A, Frost B et al. Does endoscopic ultrasound have anything to offer in the diagnosis of idiopathic acute pancreatitis? JOP 2009; 10: 143-146

[12] Vila JJ, Vicuña M, Irisarri R et al. Diagnostic yield and reliability of endoscopic ultrasonography in patients with idiopathic acute pancreatitis. Scand J Gastroenterol 2010; 45: 375-381

[13] Ardengh JC, Malheiros CA, Rahal F et al. Microlithiasis of the gallbladder: role of endoscopic ultrasonography in patients with idiopathic acute pancreatitis. Rev Assoc Med Bras 2010; 56: 27-31

[14] Rana SS, Bhasin DK, Rao C et al. Role of endoscopic ultrasound in idiopathic acute pancreatitis with negative ultrasound, computed tomography, and magnetic resonance cholangiopancreatography. Ann Gastroenterol 2012; 25: 133-137

[15] Khalid A, Peterson M, Slivka A. Secretin-stimulated magnetic resonance pancreaticogram to assess pancreatic duct outflow obstruction in evaluation of idiopathic acute recurrent pancreatitis: a pilot study. Dig Dis Sci 2003; 48: 1475-1481

[16] Testoni PA, Mariani A, Curioni S et al. MRCP-secretin test-guided management of idiopathic recurrent pancreatitis: long-term outcomes. Gastrointest Endosc 2008; 67: 1028-1034

[17] Mariani A, Arcidiacono PG, Curioni S et al. Diagnostic yield of ERCP and secretin-enhanced MRCP and EUS in patients with acute recurrent pancreatitis of unknown aetiology. Dig Liver Dis 2009; 41: 753758

[18] Ortega AR, Gómez-Rodríguez R, Romero M et al. Prospective comparison of endoscopic ultrasonography and magnetic resonance cholangiopancreatography in the etiological diagnosis of "idiopathic" acute pancreatitis. Pancreas 2011; 40: 289-294

[19] Thevenot A, Bournet B, Otal P et al. Endoscopic ultrasound and magnetic resonance cholangiopancreatography in patients with idiopathic acute pancreatitis. Dig Dis Sci 2013; 58: 2361-2368

[20] Sahai AV, Zimmerman M, Aabakken L et al. Prospec- tive assessment of the ability of endoscopic ultrasound to diagnose, exclude, or establish the severity of chronic pancreatitis found by endoscopic retrograde cholangiopancreatography. Gastrointest Endosc 1998; 48 : $18-25$

[21] Axon AT, Classen M, Cotton PB et al. Pancreatography in chronic pancreatitis: international definitions. Gut 1984; 25: 1107-1112

[22] Lee SP, Maher K, Nicholls JF. Origin and fate of biliary sludge. Gastroenterology 1988; 94: 170-176

[23] Janowitz P, Kratzer W, Zemmler T et al. Gallbladder sludge: spontaneous course and incidence of complications in patients without stones. Hepatology 1994; 20: 291-294 
[24] Ros E, Navarro S, Bru C et al. Occult microlithiasis in 'idiopathic' acute pancreatitis: prevention of relapses by cholecystectomy or ursodeoxycholic acid therapy. Gastroenterology 1991; 101: 1701-1709

[25] Dahan P, Andant C, Lévy P et al. Prospective evaluation of endoscopic ultrasonography and microscopic examination of duodenal bile in the diagnosis of cholecystolithiasis in 45 patients with normal conventional ultrasonography. Gut 1996; 38: 277-281

[26] Vila JJ, Arín A, Borobio E et al. Prospective double blinded comparison of endosonography versus microscopic bile examination for diagnosing microlithiasis in patients with biliary colic or idiopathic acute pancreatitis [Abstract]. Endoscopy 2007; 39: A281
[27] Saraswat VA, Sharma BC, Agarwal DK et al. Biliary microlithiasis in patients with idiopathic acute pancreatitis and unexplained biliary pain: response to therapy. J Gastroenterol Hepatol 2004; 19: 1206 1211

[28] Ko CW, Schulte S], Lee SP. Biliary sludge is formed by modification of hepatic bile by the gallbladder mucosa. Clin Gastroenterol Hepatol 2005; 3: 672-678

[29] Wallace MB, Hawes RH, Durkalski V et al. The reliability of EUS for the diagnosis of chronic pancreatitis: interobserver agreement among experienced endosonographers. Gastrointest Endosc 2001; 53: 294299 